\title{
BURST-BY-BURST ADAPTIVE DECISION FEEDBACK EQUALIZED TCM, TTCM, BICM AND BICM-ID
}

\author{
S. X. Ng, C. H. Wong, ${ }^{1}$ L. Hanzo \\ Dept. of Electronics and Computer Science, \\ University of Southampton, SO17 1BJ, UK. \\ Tel: +44-23-8059 3125, Fax: +44-23-80594508 \\ Email:11h@ecs.soton.ac.uk \\ http://www-mobile.ecs.soton.ac.uk
}

\begin{abstract}
Decision Feedback Equalizer (DFE) aided wideband Burst-by-Burst (BbB) Adaptive Trellis Coded Modulation (TCM), Turbo Trellis Coded Modulation (TTCM), Bit-Interleaved Coded Modulation (BICM) and BICM with Iterative Decoding (BICM-ID) are proposed and characterised in performance terms over the COST 207 Typical Urban (TU) wideband fading channel. These schemes are evaluated using a practical modem mode switching regime. System I represents schemes without channel interleaving, while System II invokes channel interleaving over four transmission bursts. A substantially improved Bit Per Symbol (BPS) and Bit Error Rate (BER) performance was achieved by System II in comparison to System I. BbB-adaptive TTCM was found to give the best performance, when aiming for a target BER of below $0.01 \%$.
\end{abstract}

\section{INTRODUCTION}

The radio spectrum is a scarce resource. Therefore, one of the most important objectives in the design of a digital cellular system is the efficient exploitation of the available spectrum in order to accommodate the ever-increasing traffic demands. Trellis Coded Modulation (TCM) [1], which is based on combining the functions of coding and modulation, is a bandwidth efficient scheme that has been widely recognized as an excellent error control technique suitable for applications in mobile communications. Turbo Trellis Coded Modulation (TTCM) [2] is a more recent channel coding scheme that has a structure similar to that of the family of power efficient binary turbo codes [3], but employs TCM codes as component codes. TCM and TTCM schemes invoked Set-Partitioning (SP) based signal labeling, in order to achieve a higher Euclidean distance between the unprotected bits of the constellation. By contrast, parallel trellis transitions are associated with the unprotected data bits, which reduces the decoding complexity. In our TCM and TTCM schemes, random symbol interleavers were utilised for both the turbo interleaver and the channel interleaver.

Another powerful coded modulation scheme utilising bit-based channel interleaving in conjunction with Gray signal labeling, which is referred to as Bit-Interleaved Coded Modulation (BICM), was proposed in [4]. It combines conventional convolutional codes with several independent bit interleavers, in order to increase the associated diversity order. With the aid of bit interleavers, the code's diversity order can be increased to the binary Hamming distance of a code, and the number of parallel bit-interleavers equals the number of coded bits in a symbol [4]. The performance of BICM is better, than that of TCM over uncorrelated (or perfectly interleaved) fading channels, but worse than that of TCM in Gaussian channels due to the reduced Euclidean distance imposed by the associated "random modulation" inherent in a bit-interleaved scheme [4]. Recently, iteratively decoded BICM using SP based signal labeling, referred to as BICM-ID has also been proposed $[5,6]$. The philosophy of BICM-ID is to increase the Euclidean distance of the BICM code and to exploit the full advantage of bit interleaving by a simple iterative decoding technique.

However, the above fixed mode transceivers failed to exploit the time varying nature of the mobile radio channels. By contrast, in BbB-adaptive schemes [7] a higher order modulation mode is employed, when the instantaneous estimated channel quality is high in order to increase the number of Bits Per Symbol (BPS) transmitted and conversely, a more robust lower order modulation mode is employed, when the instantaneous channel quality is low, in order to improve the mean Bit Error Rate (BER) performance. Uncoded adaptive schemes [7] and coded adaptive schemes [8] have been investigated for transmissions over narrowband fading channels. Finally, a turbo coded wideband adaptive scheme assisted by a Decision Feedback Equalizer (DFE) was investigated in [9].

In our practical approach, transmitter A obtains the channel quality estimate generated by receiver B upon receiving the transmission of transmitter B. In other words, the modem mode required by receiver $B$ for maintaining a certain target integrity is superimposed on the transmission burst of transmitter B. Hence a delay of one transmission burst duration is incurred.

Over wideband fading channels the DFE employed will eliminate most of the intersymbol interference (ISI). Consequently, the mean-squared error (mse) at the output of the DFE can be calculated and used as the channel quality metric invoked to switch the modulation modes [7]. This ensures that the performance is optimised by employing equalization and BbB-adaptive coded modulation jointly, in order to combat the signal power fluctuations and the ISI of the wideband channels. 


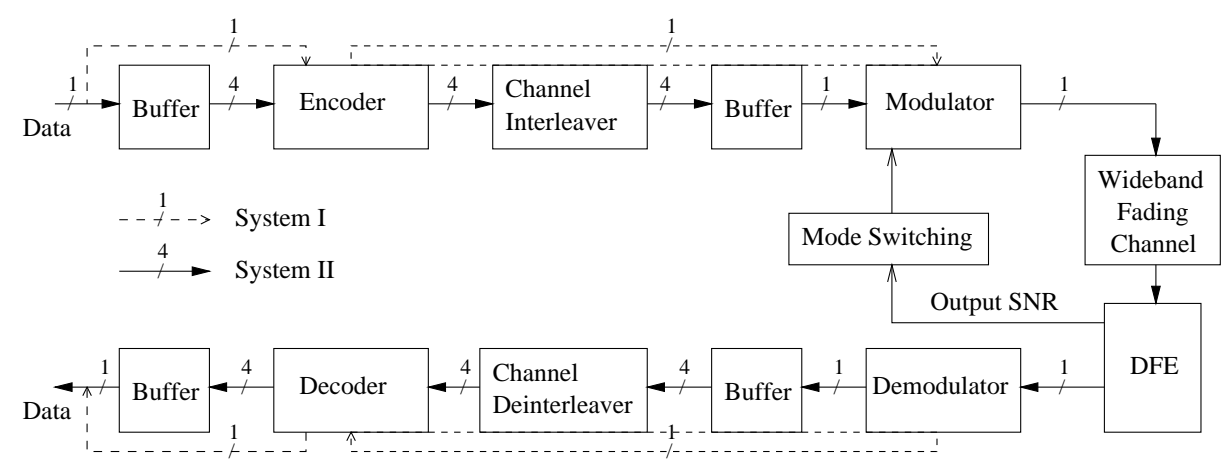

Figure 1: The non-interleaved System I and System II using a channel interleaver length of 4 TDMA/TDD bursts. In System II the equalizer's output SNR of the $4^{t h}$ burst is used to select a suitable modulation mode and fed back to the transmitter on the reverse link burst.

\section{SYSTEM OVERVIEW}

The block diagram of the BbB-adaptive coded modulation System I, where no channel interleaving is used, can be seen in Figure 1, the buffers and channel interleavers are by-passed. Transmitter A extracts the modulation mode required by receiver $B$ from the reverse-link transmission burst in order to adjust the adaptive modulation mode suitable for the instantaneous channel quality. This incurs one TDMA/TDD frame delay between estimating the actual channel condition at receiver $\mathrm{B}$ and the selected modulation mode of transmitter A. We invoke four encoders, each adding one parity bit to each information symbol, yielding a coding rate of $1 / 2$ in conjunction with the modulation modes of $4 \mathrm{QAM}$, and a rate of $2 / 3$ for $8 \mathrm{PSK}, 3 / 4$ for 16QAM and 5/6 for 64QAM.

The design of coded modulation schemes for fading channels relies on the time and space diversity provided by the associated encoder [10]. Diversity may be achieved by repetition coding (which reduces the effective data rate), spacetime coded multiple transmitter/receiver structures (which increases cost and complexity) or by simple interleaving (which induces latency). Here, we invoke interleaving to achieve extra diversity in System II. Explicitly, Figure 1 also shows the block diagram of System II, where channel interleaving over four transmission bursts is utilised, in order to disperse the bursty channel errors. Hence, the coded modulation module assembles four consecutive bursts using an identical modulation mode, so that they could be interleaved using a random channel interleaver without the need of adding dummy bits. Then, these four-burst packets are transmitted to the receiver. Once the receiver has received the $4^{t h}$ burst, the equalizer's output SNR estimated for this most recent burst is used to choose a suitable modulation mode. The selected modulation mode is fed back to the transmitter on the reverse link burst. Upon receiving the modulation mode required by receiver $\mathrm{B}$ (after one TDMA frame delay), the coded modulation module assembles four bursts of data from its input buffer for coding and interleaving, which are then stored in the output buffer ready for the next four bursts' transmission. Thus the first transmission burst experiences one TDMA/TDD frame delay and the fourth transmission burst exhibits four frame delay, which is the worst-case scenario.

The complexity of the coded modulation schemes is compared in terms of the number of decoding states and the number of decoding iterations. For a TCM or BICM code of memory $M$, the corresponding complexity is proportional to the number of decoding states $S=2^{M}$. Since TTCM schemes invoke two component TCM codes, a TTCM code with $t$ iterations and using an $S$-state component code exhibits a complexity proportional to $2 . t . S$ or $t .2^{M+1}$. As for the BICM-ID schemes, only one decoder is used, but the demodulator is invoked in each decoding iteration. However, the complexity of the demodulator is assumed to be insignificant compared to that of the decoder. Hence, a BICM-ID code with $t$ iterations using an $S$-state code exhibits a complexity proportional to $t . S$ or $t .2^{M}$. A component TCM (BICM) code memory of 3 was used for the TTCM (BICM-ID) scheme. The number of iterations for TTCM (BICM-ID) was fixed to 4 (8) and hence the iterative scheme exhibited a similar decoding complexity to the TCM (BICM) code of memory 6 in terms of the number of coding states.

The fixed-mode coded modulation schemes that we invoked in our BbB-adaptive schemes are Ungerboeck's TCM [1], Robertson's TTCM [2], Zehavi's BICM [4] and Li's BICM-ID using soft-decision feedback [6]. In order to reduce the decoding memory, the BICM and BICM-ID codes used in 64QAM were obtained by puncturing the rate- $1 / 2$ codes following the approach of [11]. Soft decision trellis decoding utilizing the Log-Maximum A Posteriori (Log-MAP) algorithm [12] was invoked for decoding. The Log-MAP algorithm is a numerically stable version of the MAP algorithm operating in the log-domain, in order to reduce its complexity and to mitigate the numerical problems associated with the MAP algorithm [13].

The multi-path channel model is characterized by its discretised symbol-spaced COST207 Typical Urban (TU) channel impulse response [14], as shown in Figure 2. Each path is faded independently according to a Rayleigh distribution and the corresponding normalised Doppler frequency is $3.25 \times 10^{-5}$, the system's Baud rate is $2.6 \mathrm{MBd}$, the carrier frequency is $1.9 \mathrm{GHz}$ and the vehicular speed is $30 \mathrm{mph}$. The DFE incorporated 35 feed-forward taps and 7 feedback taps and the transmission burst structure used is shown in Figure 3. When considering a Time Division Multiple Access (TDMA)/Time Division Duplex (TDD) system of 16 slots per $4.615 \mathrm{~ms}$ TDMA frame, the transmission burst duration is $288 \mu s$, as specified in the Pan-European FRAMES proposal [15].

At the receiver, the Channel Impulse Response (CIR) 


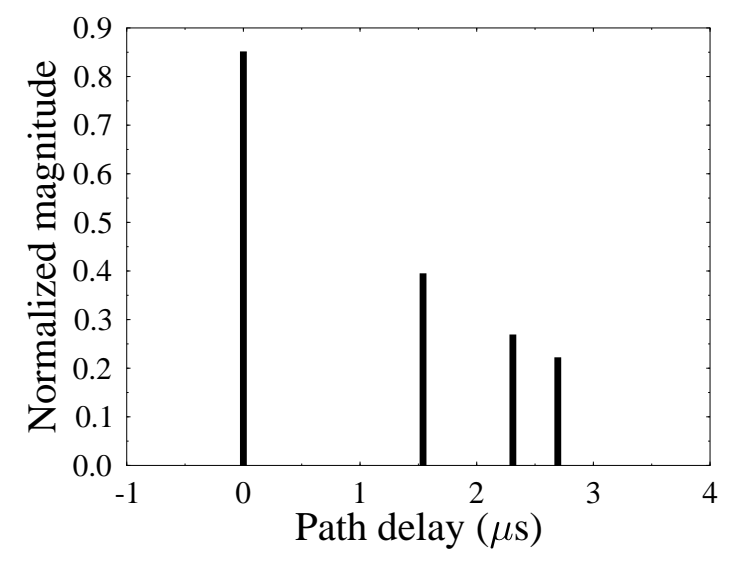

Figure 2: The impulse response of a COST 207 Typical Urban (TU) channel [14].

\begin{tabular}{|c|c|c|c|}
\hline \multicolumn{3}{|c|}{288 microseconds } \\
\hline 342 data symbols & 49 symbols & 342 data symbols & \\
\hline & & & \\
\hline Data & $\begin{array}{l}\text { Training } \\
\text { sequence }\end{array}$ & Data \\
\hline
\end{tabular}

non-spread data burst

Figure 3: Transmission burst structure of the FMA1 nonspread data as specified in the FRAMES proposal [15].

is estimated, which is then used to calculate the DFE coefficients [7]. Subsequently, the DFE is used to equalize the ISI-corrupted received signal. In addition, both the CIR estimate and the DFE feed-forward coefficients are utilized to compute the SNR at the output of the DFE. More specifically, by assuming that the residual ISI is near-Gaussian distributed and that the probability of decision feedback errors is negligible, the SNR at the output of the DFE, $\gamma_{d f e}$, is calculated as [7]:

$$
\begin{gathered}
\gamma_{d f e}=\frac{\text { Wanted Signal Power }}{\text { Residual ISI Power + Effective Noise Power }} \\
=\frac{E\left[\left|s_{k} \sum_{m=0}^{N_{f}} C_{m} h_{m}\right|^{2}\right]}{\sum_{q=-\left(N_{f}-1\right)}^{-1} E\left[\left|\sum_{m=0}^{N_{f}-1} C_{m} h_{m+q} s_{k-q}\right|^{2}\right]+N_{o} \sum_{m=0}^{N_{f}}\left|C_{m}\right|^{2}}
\end{gathered}
$$

where $C_{m}$ and $h_{m}$ denotes the DFE's feed-forward coefficients and the CIR, respectively. The transmitted signal is represented by $s_{k}$, while $N_{0}$ denotes the noise spectral density. Lastly, the number of DFE feed-forward coefficients is denoted by $N_{f}$.

\begin{tabular}{|l|l|c|c|c|}
\hline \multicolumn{2}{|l|}{ BER < 0.01 \% } & \multicolumn{3}{|c|}{ Switching Thresholds } \\
\cline { 3 - 5 } Adaptive System Type & $f_{1}$ & $f_{2}$ & $f_{3}$ \\
\hline TCM, Memory 3 & System I & 19.56 & 23.91 & 30.52 \\
& System II & 17.17 & 21.91 & 29.61 \\
\hline TCM, Memory 6 & System I & 19.56 & 23.88 & 30.07 \\
& System II & 17.14 & 21.45 & 29.52 \\
\hline TTCM, 4 iterations & System I & 19.69 & 23.45 & 30.29 \\
& System II & 16.66 & 21.40 & 28.47 \\
\hline BICM, Memory 3 & System I & 19.94 & 24.06 & 31.39 \\
\hline BICM-ID, 8 iterations & System II & 16.74 & 21.45 & 28.97 \\
\hline
\end{tabular}

Table 1: The switching thresholds were set experimentally in order to achieve a target BER of below $0.01 \%$. System I does not utilise a channel interleaver, while System II uses a channel interleaver length of 4 TDMA/TDD bursts.

The BbB-adaptive coded modem mode switching mechanism is characterised by the comparison of the equalizer's SNR, $\gamma_{d f e}$, in Equation 1 to a set of switching thresholds, as follows:

$$
\text { Mode }= \begin{cases}4 Q A M, I_{0}=684, R_{0}=1 / 2 & \text { if } \gamma \leq f_{1} \\ 8 P S K, I_{1}=2 I_{0}, R_{1}=2 / 3 & \text { if } f_{1}<\gamma \leq f_{2} \\ 16 Q A M, I_{2}=3 I_{0}, R_{2}=3 / 4 & \text { if } f_{2}<\gamma \leq f_{3} \\ 64 Q A M, I_{3}=5 I_{0}, R_{3}=5 / 6 & \text { if } \gamma>f_{3}\end{cases}
$$

where $f_{n}, n=1 \ldots 3$ are the equalizer's output SNR thresholds, while $I_{n}$ represents the random TTCM symbol-interleaver size in terms of the number of bits, which is not used for the other schemes. The switching thresholds $f_{n}$ were chosen experimentally, in order to maintain a BER of below $0.01 \%$ and these thresholds are listed in Table 1 .

The following assumptions are stipulated. Firstly, we assume that the equalizer is capable of estimating the CIR perfectly from the equaliser training sequence of Figure 3. Secondly, the CIR is time-invariant for the duration of a transmission burst, but varies from burst to burst according to the Doppler frequency, which corresponds to assuming that the CIR is slowly varying. The error propagation of the DFE will degrade the estimated performance, but the effect of error propagation is left for further study.

\section{SIMULATION RESULTS AND DISCUSSIONS}

Let us consider the BbB-adaptive TTCM scheme in order to investigate the performance of System I and System II. The BER and BPS performances of BbB-adaptive TTCM in both systems are shown in Figure 4, where we observed that the throughput of System II was superior to that of System I. Furthermore, the overall BER of System II was lower than that of System I.

Figure 5 shows the BER and BPS performance of System I for BbB-adaptive TTCM, BbB-adaptive TCM of memory 3 (which was the component code of our TTCM scheme) and BbB-adaptive TCM of memory 6. All these codes exhibited a similar performance in the BbB-adaptive schemes of System I. Even the TCM scheme of memory 3 associated with a lower complexity could give a similar BER and BPS performance to the more complex schemes. This shows that the equalizer plays a dominant role in System I, where the coded modulation schemes could not realise their full potential due to their insufficient diversity, when 


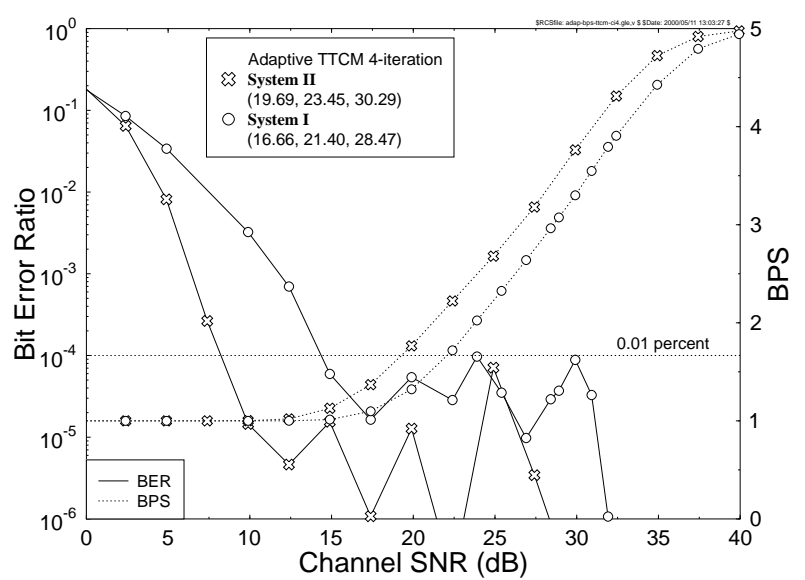

Figure 4: BER and BPS performance of BbB-adaptive TTCM in System I and in System II, over the Rayleigh fading COST207 TU channels of Figure 2 for a target BER of less than $0.01 \%$. The legends indicate the associated switching thresholds expressed in $d B$, as seen in the round brackets.



Figure 5: BER and BPS performance of BbB-adaptive TCM and TTCM without channel interleaving in System I, over the Rayleigh fading COST207 TU channels of Figure 2. The switching mechanism was characterized by Equation 2. The switching thresholds were set experimentally, in order to achieve a BER of below $0.01 \%$, as shown in Table 1.

no channel interleaver was used over the slow fading COST 207 wideband channel.

When the channel interleaver was introduced in System II, the bursty errors were more efficiently randomised. Figure 6 illustrates the BER and BPS performance of System II in conjuction with BbB-adaptive TTCM, BbBadaptive TCM of memory 3 and BbB-adaptive TCM of memory 6 . The performance of all these schemes improved in the context of System II, as compared to the corresponding schemes in System I. The TCM scheme of memory 6 had a lower BER, than TCM of memory 3, and also exhibited a small BPS improvement. As expected, TTCM had the lowest BER and also the highest BPS throughput,

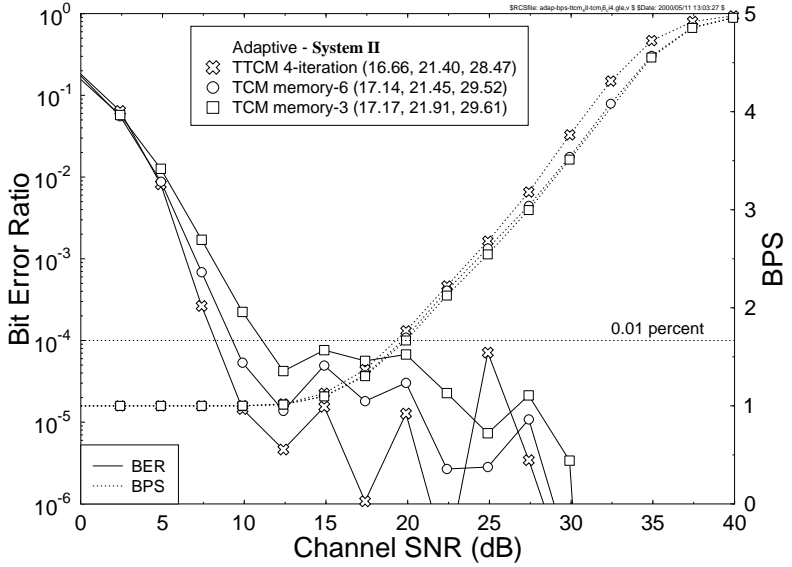

Figure 6: BER and BPS performance of BbB-adaptive TCM and TTCM using a channel interleaver length of 4 bursts, in System II over the Rayleigh fading COST207 TU channels of Figure 2. The switching mechanism was characterized by Equation 2. The switching thresholds were set experimentally, in order to achieve a BER of below $0.01 \%$, as shown in Table 1 .

compared to the other coded modulation schemes.

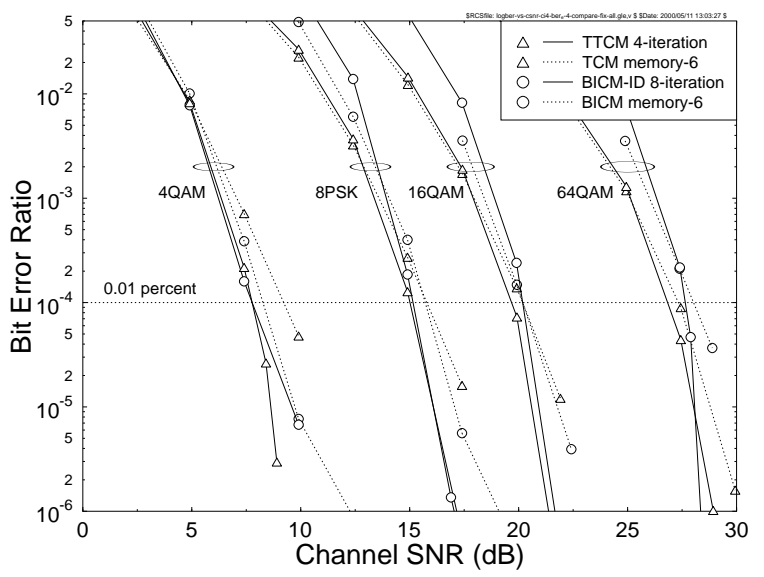

Figure 7: BER performance of the fixed modem modes of 4QAM, 8PSK, 16QAM and 64QAM utilising TCM, TTCM, BICM and BICM-ID schemes in the context of System II.

Figure 7 shows the fixed modem modes' performance for TCM, TTCM, BICM and BICM-ID in the context of System II. As illustrated in the figure, the BICM scheme performed marginally better, than the TCM scheme at a BER below $0.01 \%$, except in the 64QAM mode. Hence, BbBadaptive BICM is also expected to perform better, than BbB-adaptive TCM in the context of System II, when a target BER of less than $0.01 \%$ is desired. This is because when the channel interleaver depth is sufficiently high, the diversity gain of the BICM's bit-interleaver is higher, than that of the TCM's symbol-interleaver [4].

Figure 8 compares the BbB-adaptive BICM and TCM schemes in the context of System I, i.e. without chan- 


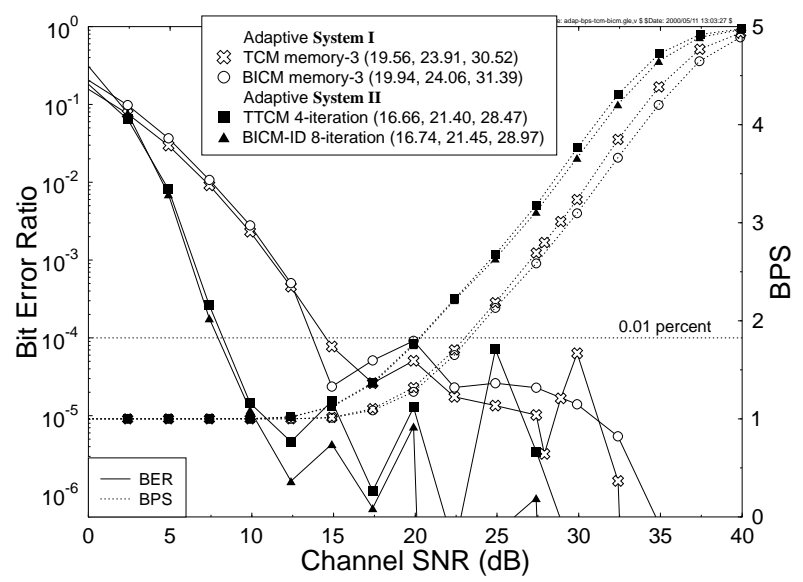

Figure 8: BER and BPS performance of the BbB-adaptive TCM/BICM System I, using memory 3 codes and that of the BbB-adaptive TTCM/BICM-ID System II, over the Rayleigh fading COST207 TU channels of Figure 2. The switching mechanism was characterized by Equation 2. The switching thresholds were set experimentally, in order to achieve a BER of below $0.01 \%$, as shown in Table 1 .

nel interleaving, although the BICM scheme invoked an internal bit-interleaver of one burst memory. As it can be seen from the figure, BbB-adaptive TCM exhibited a better BPS throughput and BER performance than BICM, due to the insufficient channel interleaving depth of the BICM scheme in our slow fading wideband channels. As observed in Figure 7, BICM-ID had the worst performance at low SNRs in each modulation mode compared to the other coded modulation schemes. However, it exhibited a steep slope and therefore at high SNRs it approached the performance of the TTCM scheme. The BbB-adaptive BICM-ID and TTCM schemes were compared in Figure 8 in the context of System II. BbB-adaptive TTCM exhibited a better BPS throughput, than BbB-adaptive BICM-ID, since TTCM had a better performance in the fixed modem modes at BERs below $0.01 \%$. However, BbB-adaptive BICM-ID exhibited a lower BER, than BbB-adaptive TTCM due to the high steepness of the BICM-ID BER curves in the fixed modem modes.

\section{CONCLUSION}

In this contribution, BbB-adaptive TCM, TTCM, BICM and BICM-ID were proposed for wideband fading channels both with and without channel interleaving and they were characterised in performance terms over the COST 207 TU fading channels. When observing the associated BPS curves, BbB-adaptive TTCM exhibited up to $2.5 \mathrm{~dB}$ SNR-gain for a channel interleaver length of 4 bursts in comparison to the non-interleaved scenario, as evidenced by Figure 4. Upon comparing the BPS curves, BbB-adaptive TTCM also exhibited up to $0.7 d B$ SNR-gain compared to BbB-adaptive TCM of the same complexity in the context of System II for a target BER of less than $0.01 \%$, as shown in Figure 6. Finally, BbB-adaptive TCM performed better than BbB-adaptive BICM in System I, while BbB-adaptive BICM-ID was marginally worse, than BbB- adaptive TTCM in System II, as seen in Figure 8.

\section{ACKNOWLEDGEMENTS}

The financial support of the European Union and the EP$\mathrm{SRC}, \mathrm{UK}$ is gratefully acknowledged.

\section{REFERENCES}

[1] G. Ungerboeck, "Channel coding with multilevel/Phase signal," IEEE Transactions on Information Theory, vol. 28, pp. 55-67, January 1982.

[2] P. Robertson, T. Wörz, "Bandwidth-Efficient Turbo TrellisCoded Modulation Using Punctured Component Codes," IEEE Journal on Selected Areas in Communications, vol. 16, pp. 206-218, February 1998.

[3] C. Berrou, A. Glavieux and P. Thitimajshima, "Near Shannon Limit Error-Correcting Coding and Decoding : Turbo Codes," in Proceedings, IEEE International Conference on Communications, pp. 1064 - 1070, 1993.

[4] E. Zehavi, "8-PSK trellis codes for a Rayleigh fading channel," IEEE Transactions on Communications, vol. 40, pp. 873 - 883, May 1992.

[5] X. Li, J. A. Ritcey, "Trellis-Coded Modulation with Bit Interleaving and Iterative Decoding," IEEE Journal on Selected Areas in Communications, vol. 17, No. 4, April 1999.

[6] X. Li, J. A. Ritcey, "Bit-interleaved coded modulation with iterative decoding using soft feedback," IEE Electronics Letters, vol. 34, No. 10, pp. 942 - 943, May 1998.

[7] C. H. Wong and L. Hanzo, "Upper-Bound Performance of a Wide-Band Adaptive Modem," IEEE Transactions on Communications, vol. 48, pp. 367 - 369, March 2000.

[8] S. G. Chua and A. Goldsmith, "Adaptive Coded Modulation for Fading Channels," IEEE Transactions on Communications, vol. 46, pp. 595 - 602, May 1998.

[9] C. H. Wong, T. H. Liew and L. Hanzo, "Burst-by-Burst Turbo Coded Wideband Adaptive Modulation with Blind Modem Mode Detection," in Proceedings of 4th ACTS Mobile Communications Summit 1999, Sorrento, Italy, pp. 303-308, 8 - 11 June 1999.

[10] D. Divsalar and M. K. Simon, "The design of trellis coded MPSK for fading channel: Performance criteria," IEEE Transactions on Communications, vol. 36, pp. 1004-1012, September 1988.

[11] L. H. C. Lee, "New Rate-Compatible Puncture Convolutional Codes for Viterbi Decoding," IEEE Transactions on Communications, vol. 42, pp. 3073 - 3079, December 1994.

[12] P. Robertson, E. Villebrum and P. Hoeher, "A Comparison of Optimal and Sub-Optimal MAP Decoding Algorithms Operating in Log Domain," in Proceedings of the International Conference on Communications , pp. 1009-1013, June 1995.

[13] L. R. Bahl, J. Cocke, F. Jelinek and J. Raviv, "Optimal Decoding of Linear Codes for Minimizing Symbol Error Rate," IEEE Transactions on Information Theory, vol. 20, pp. 284-287, March 1974.

[14] Office for Official Publications of the European Communities, Luxembourg, COST 207: Digital land mobile radio communications, Final Report, 1989.

[15] A. Klein, R. Pirhonen, J.Sköld and R.Suoranta, "FRAMES Multiple Access Mode 1 - Wideband TDMA with and without spreading," in Proceedings of PIMRC'97, pp. 37-41, 1997. 